\title{
Combination Low Dose Cyclosporin A and Steroid Therapy in Chronic Intraocular Inflammation
}

\author{
H. M. A. TOWLER, P. H. WHITING* and J. V. FORRESTER \\ Aberdeen
}

\begin{abstract}
Summary
Thirteen patients with chronic intraocular inflammation which had not been adequately controlled with oral prednisolone entered into an open study of low-dose Cyclosporin A (mean $4.1 \mathrm{mg} / \mathrm{kg} /$ day) combined when required with oral prednisolone (15 $\mathrm{mg}$ prednisolone per day or less). The mean duration of treatment was 26 months (range 8-44) over a mean follow-up period of 29 months (range 8-49). Visual acuity improved overall in ten patients, and remained stable in three. Six patients have completed a course of Cyclosporin A therapy, and four of these patients have retained their visual improvement. Two have returned to the pretreatment level of vision. Only one patient has required conversion to alternative immunosuppressive therapy. The mean serum creatinine concentration had increased significantly by $26 \%$ after six months $(p<0.05)$ and $32 \%$ after one year $(p<0.01)$ but remained stable during the subsequent 18 months. Four patients developed hypertension requiring hypotensive therapy. On cessation of CsA treatment, the mean serum creatinine concentration fell to the upper reference limit. The elevation of serum creatinine concentration was significantly higher in patients who either developed hypertension during Cyclosporin A therapy or who were previously known to be hypertensive. Patients with nephrotoxicity were significantly older than those in whom serum creatinine concentration remained within the reference range. Lithium clearance studies showed evidence of proximal renal tubular dysfunction which was partially reversible on dose reduction or withdrawal.
\end{abstract}

Chronic intraocular inflammation ${ }^{1}$ is a significant and serious cause of visual handicap in young and middle-aged adults. 'Iritis' accounted for approximately the same number of blind registrations as retinal detachment under the age of 64 years in England and Wales in 1969-76 (314 versus 343 respectively), and was the certified cause of $2.4 \%$ and $2.7 \%$ of partial sight and blind registration respectively for the same period. ${ }^{2}$
These figures underestimate the contribution that chronic intraocular inflammation makes to visual morbidity in the $\mathrm{UK}$ and, in the United States, approximately $10 \%$ of visual handicap is attributed to uveitis. ${ }^{3}$ Treatment with systemic corticosteroids is often required to control sight threatening inflammation and, failing this, more powerful immunosuppressive therapy may be necessary. Disease activity may recur on dose reduction

From: Departments of Ophthalmology and Clinical Biochemistry*, University of Aberdeen, Medical School, Foresterhill, Aberdeen AB9 2ZD.

Correspondence to Professor JV Forrester, Department of Ophthalmology, Medical School, Foresterhill, Aberdeen AB9 2ZD.

Presented at the Oxford Ophthalmological Congress, July 1989. 
or side effects may preclude high dose steroid administration for prolonged periods, necessitating introduction of alternative therapy.

The inhibitory effect of Cyclosporin A (CsA) therapy on intraocular inflammation, first shown in experimental autoimmune uveitis in Lewis rats, ${ }^{4}$ has been confirmed in a number of clinical studies. ${ }^{3,5-8}$ However, widespread acceptance of CsA treatment has not been achieved due to considerable anxiety about the drug's nephrotoxicity, ${ }^{9-12}$ uncertainty about the optimum duration of treatment, and questions about its long-term safety, ${ }^{13}$ though the risk of neoplasia with doses of or less than $5 \mathrm{mg} / \mathrm{kg} /$ day is considered negligible.

We previously reported the successful use of low dose CsA therapy for a mean period of 17 months in nine patients with chronic intraocular inflammation ${ }^{14}$ and now present the results of combined CsA and steroid therapy in a larger group for a mean period of 29 months.

\section{Patients and Methods \\ Study Group}

Clinical details of the study group are shown in Table I. Pars planitis and retinal vasculitis of varying aetiology, usually associated with cystoid macular oedema, were the most common diseases for which CsA therapy was indicated. All patients had either failed to respond adequately to oral prednisolone or had relapsed when the dose of prednisolone was reduced below $20 \mathrm{mg}$ per day. Steroid treatment had been continued for at least one year prior to the introduction of CsA therapy, when all patients were receiving at least $20 \mathrm{mg}$ prednisolone daily. This was quickly reduced to a dose of $10-15 \mathrm{mg}$ per day and continued for at least three months after achieving a stable maintenance dose of CsA, though four patients were subsequently able to discontinue steroids. The mean patient age was 40.7 years, mean duration of treatment 26.1 months, and mean duration of follow up was 29 months after the introduction of CsA therapy. The clinical, visual, and biochemical parameters monitored before, during, and after the period of CsA treatment were described previously. ${ }^{14}$ The intraocular inflammation scoring system was modified to include the assessment of vitreous haze with the binocular indirect ophthalmoscope (BIO).${ }^{15}$ In addition to assessment of renal function by measurement of serum creatinine and creatinine clearance, renal tubular function was assessed using the lithium clearance method of Thomsen. ${ }^{16} \mathrm{~A}$ more detailed analysis of the renal function changes will be published elsewhere.

\section{Cyclosporin A Regimen}

Treatment was commenced as a single oral dose of $5 \mathrm{mg} / \mathrm{kg} /$ day. The first four patients had been commenced on $10 \mathrm{mg} / \mathrm{kg} / \mathrm{day}$ but this dose had produced high blood levels and the lower dose was used thereafter. The maintenance dose, taken as a single oral dose in the evening, was thereafter adjusted to achieve the lowest dose compatible with control of intraocular inflammation, minimum nephrotoxicity, and a trough whole blood level of $300-800 \mu \mathrm{g} / \mathrm{l}$. Blood levels of CsA were measured with a radioimmunoassay kit provided by Sandoz, Ltd, initially using a polyclonal anti-CsA antibody which could not distinguish between the parent compound and certain of its metabolites. A monoclonal anti-CsA antibody was subsequently employed, which has a lower recommended therapeutic range of $90-360 \mu \mathrm{g} / \mathrm{l}$. CsA levels were measured at weekly intervals until stable and monthly therafter. Patients were initially seen weekly following induction of CsA treatment in hospital and thereafter on a monthly basis or more often if clinically indicated. CsA therapy was continued for a minimum of twelve months before stopping treatment, which was achieved by dose reduction of $50 \mathrm{mg}$ per month. Statistical analysis was by paired Student $t$ test or Mann-Whitney U test for non-parametric data.

\section{Results}

After one year of therapy, visual acuity had improved in eleven eyes, remained the same in four, and deteriorated in three. Visual improvement was maintained after two years (Fig. 1) and at the most recent review. Reduction of CsA dose was associated with increasing inflammatory activity in two patients, which was subsequently controlled by returning to the previous maintenance dose. The 
Table I Study group details

\begin{tabular}{ccclccc}
\hline Patient & Sex & Age & Diagnosis & $\begin{array}{c}\text { Maintenance } \\
\text { dose of CsA } \\
\text { (mglkg) }\end{array}$ & $\begin{array}{c}\text { Duration of } \\
\text { CsA therapy } \\
\text { (months) }\end{array}$ & $\begin{array}{c}\text { Follow up } \\
\text { (months) }\end{array}$ \\
\hline 1 & F & 29 & Retinal pigment epitheliitis & 4.4 & 29 & 32 \\
2 & M & 37 & Retinal vasculitis & 3.75 & 39 & 41 \\
3 & M & 41 & Sarcoidosis, retinal vasculitis & 3.75 & 41 & 46 \\
4 & F & 58 & Pars planitis, CMO & 3.0 & 31 & 36 \\
5 & M & 64 & Pars planitis, CMO & 3.4 & 14 & 22 \\
6 & F & 63 & Birdshot choroidopathy, CMO & 4.0 & 27 & 37 \\
7 & M & 46 & Behçet's disease, scleritis, CMO & 2.75 & 26 & 26 \\
8 & M & 48 & Sarcoidosis, retinal vasculitis & 3.7 & 40 & 49 \\
9 & M & 18 & Pars planitus, CMO & 4.8 & 44 & 44 \\
10 & F & 59 & Birdshot choroidopathy & 4.0 & 16 & 16 \\
11 & F & 10 & Panuveitis, ANF + JCA & 5.5 & 16 & 16 \\
12 & M & 24 & Pars planitis, CMO & 4.9 & 8 & 8 \\
13 & F & 32 & Behçet's disease, retinal vasculitis & 4.7 & 8 & 8 \\
Mean & & 40.7 & & 4.1 & 26.1 & 29 \\
SD & & 17.6 & & 0.8 & 12.7 & 14 \\
\hline
\end{tabular}

$\mathrm{CMO}=$ Cystoid Macular Oedema. $\mathrm{JCA}=$ Juvenile Chronic Arthritis.

development of nephrotoxicity and hypertension in one patient necessitated conversion to azathioprine therapy although control of his intraocular inflammation had been achieved. Of the six patients who have stopped CsA treatment, five eyes showed improvement compared with pretreatment vision, four have returned to pretreatment levels, and one has deteriorated. Four eyes were excluded from analysis for the following reasons: one blind from rhegmatogenous retinal detachment, one densely amblyopic, and

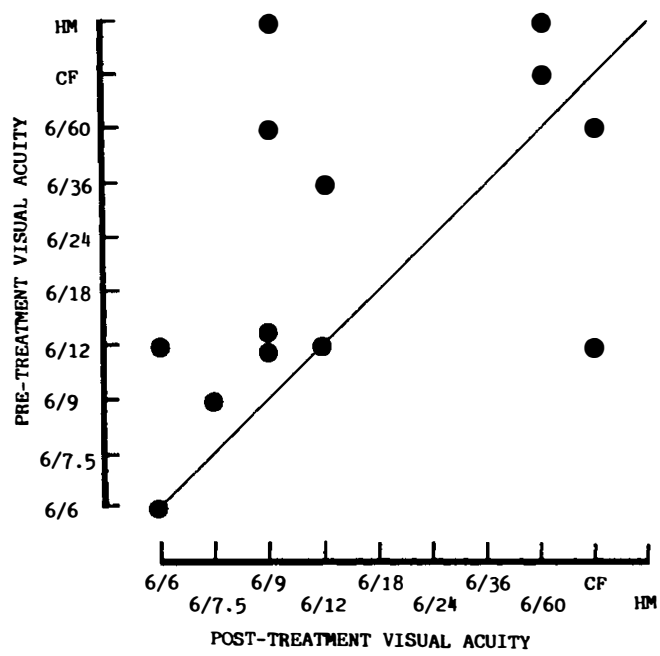

Fig. 1. Visual outcome after two years of low dose CsA therapy. two eyes with visual acuity of $6 / 5$ and no evidence of any intraocular inflammation throughout the study (patients 7 and 11).

Improvement in visual acuity was associated with a variety of clinical changes. Clearing of vitreous infiltrate with subsequent fall in $\mathrm{BIO}$ grade was the most constant finding. Vision also improved due to resolution of macular oedema or to control of retinal vasculitis (Figs. 2 and 3). Deteriorating visual acuity in three patients was due to progression of cataract in one, the development of macular ischaemia secondary to retinal vasculitis in another, and to the development and progression of a subretinal neovascular membrane below the papillomacular bundle and fovea in the third. In the latter patient, the deterioration occurred despite good control of her intraocular inflammation.

The side effects of treatment are shown in Table II. The most common toxic effect was a significant rise in mean serum creatinine concentration by $26 \%$ of the pretreatment level after six months of treatment, by $32 \%$ after one year, and thereafter remaining stable for the next 18 months. Three months after cessation of CsA therapy in six patients, the mean serum creatinine concentration fell to the upper limit of the reference range. The elevation of serum creatinine concentration was significantly greater in five patients with hypertension, one of whom was previously 

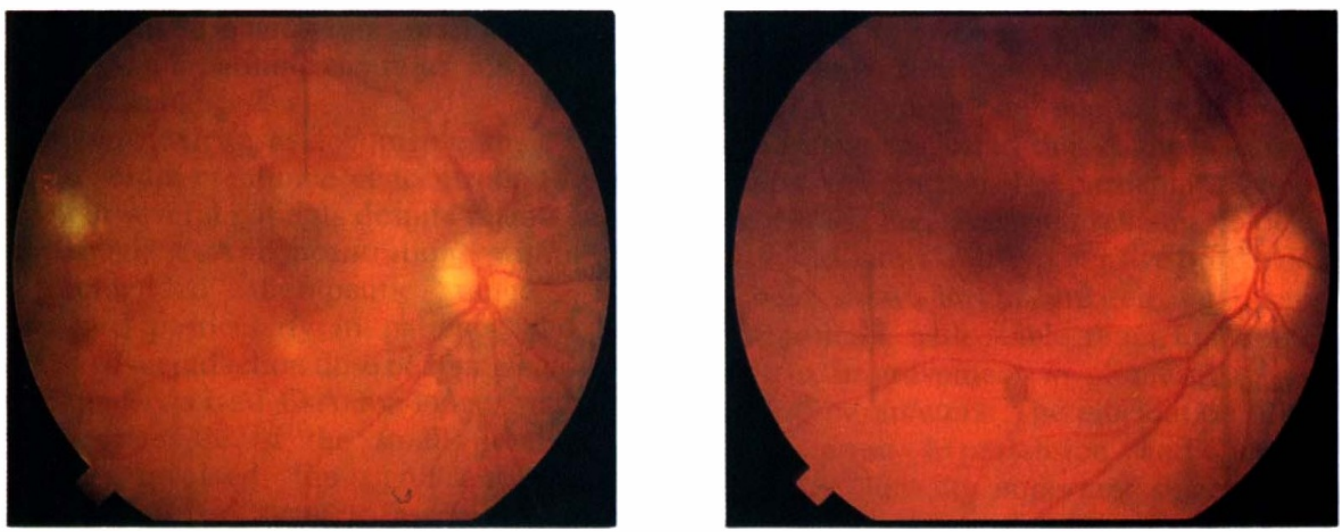

Figs. 2 and 3. Pre-treatment (Fig. 2) and Post-treatment (Fig. 3). Resolution of retinal vasculitis and clearing of vitreous haze after $\operatorname{Cs} A$ therapy.

known to be hypertensive and the other four who developed hypertension requiring treatment while taking CsA (Fig. 4). The mean age of patients who developed nephrotoxicity was significantly greater than those with normal serum creatinine concentration $(53.6 \pm 10.1$ vs $25.7 \pm 10.9$ respectively, $\mathrm{p}<0.001)$. Hypertensive patients were not significantly older than normotensive patients $(40.6 \pm 9.1$ vs $35.1 \pm 19.8$ respectively). In a group of six patients who had pretreatment lithium and creatinine clearance studies (Table III), there was a $15 \%$ reduction of creatinine clearance (p NS), a $64 \%$ reduction of lithium clearance $(\mathrm{p}<0.01)$ and a $61 \%$ reduction of fractional excretion of lithium (FELi, $\mathrm{p}<0.01)$ after six months of CsA treatment. After twelve months, the creatinine clearance was little changed, and the lithium clearance and FELi had recovered to $57 \%$ and $72 \%$ respectively of their pretreatment value but were still significantly reduced.

\section{Discussion}

The results of this open study confirm the visual benefits of combination low dose CsA and steroid therapy over a sustained period in patients with chronic intraocular inflammation which was difficult to control with oral steroids alone. The improvement in visual acuity seen after the first year of combination therapy has been maintained in those patients who continued treatment for two years or more. After withdrawal of CsA therapy, the majority of patients maintained their improvement of visual acuity or showed no visual deterioration in comparison with their pretreatment visual acuity. It is well recognised that patients with chronic intraocular inflammation frequently develop permanent visual handicap due to irreversible macular damage due, for example, to chronic cystoid macular oedema or retinal vascular occlusion, so that preservation of visual acuity over a period of two to three years may be considered a successful clinical result. Furthermore, visual deterioration may occur despite control of intraocular inflammation due to progression of other pathological disease processes, such as the development of subretinal neovascularisation involving the papillomacular bundle and fovea as occurred in one of the patients with birdshot choroidopathy in this report.

This study does, however, emphasise that nephrotoxicity and hypertension may occur even with a low dose regime and that these indices of toxicity need to be closely moni-

Table II CsA toxicity (numbers affected in parentheses)

\begin{tabular}{ll}
\hline Major & Minor \\
\hline Nephrotoxicity (10) & Hirsutism (6) \\
Hypertension (4) & Hypomagnesaemia (4) \\
Hepatotoxicity (0) & Taste disturbance (4) \\
Neoplasia (0) & Rashes (3) \\
& Paraesthesiae (2) \\
& Gingivitis (2) \\
& Cramps (11) \\
& Nail abnormality (1) \\
\hline
\end{tabular}




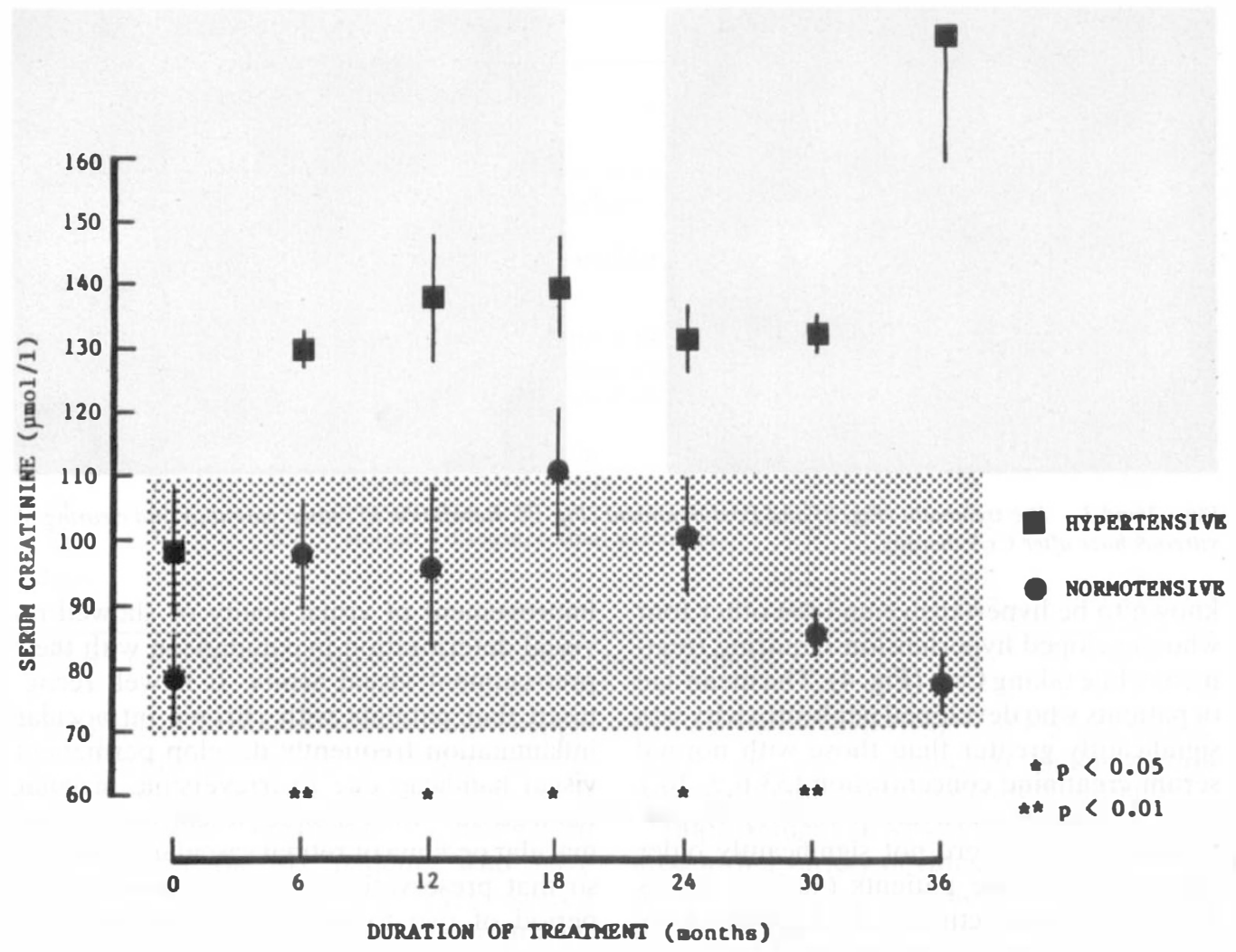

Fig. 4. Serum creatinine changes (Mean $\pm S E M$ ) in relation to blood pressure during low-dose CsA therapy (reference range shaded).

tored in all patients receiving CsA therapy. The $32 \%$ elevation of mean serum creatinine concentration and $31 \%$ incidence of sustained hypertension requiring treatment after the first twelve months of CsA therapy are of similar magnitude to other reports on the use of CsA in uveitis, ${ }^{6,9,17,18}$ all of which employed a mean maintenance dose of $10 \mathrm{mg} / \mathrm{kg} /$ day. These observations suggest that hypertension and nephrotoxicity are not simply related to the total cumulative dose of CsA as was suggested from early reports of CsA use in organ transplantation where much higher dose regimes were used. The mean serum creatinine concentration is a simple and reliable biochemical indicator of nephrotoxicity but it may not be sufficiently sensitive to detect early changes in renal tubular function which may be relevant to the development of interstitial changes and chronic irreversible nephrotoxicity. The changes in proximal renal tubule function shown in this study by the clearance and fractional excretion of lithium indicate that tubular dysfunction can occur

Table III Effect of Cyclosporin A on renal function

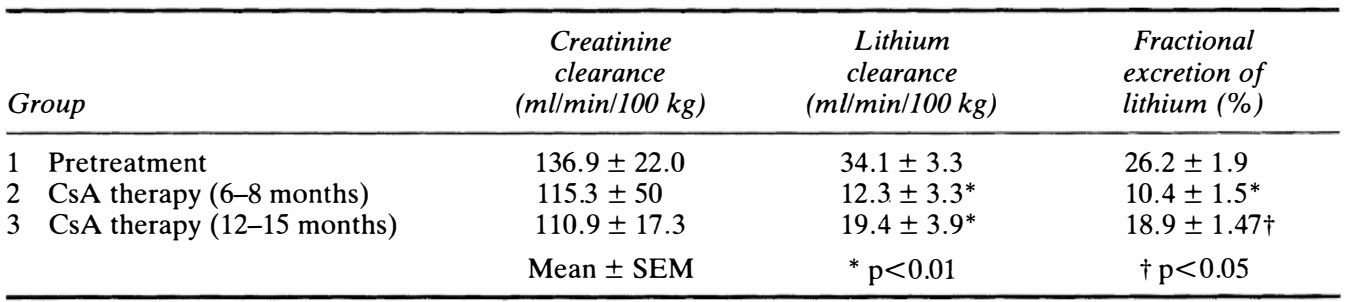


even when the mean serum creatinine concentration and creatinine clearance have not significantly changed.

Nephrotoxicity, as documented by a rise in mean serum creatinine concentration developed in several patients despite maintenance of trough CsA concentrations within the recommended therapeutic range. This occurred particularly in patients who had received an induction dose of $10 \mathrm{mg} / \mathrm{kg} /$ day or who had received CsA for longer than two years. Because of the small numbers of patients involved, the significance of this observation is difficult to assess but it may support the suggestion of Klintmalm et al. ${ }^{11}$ that the cumulative dose in the first six months of CsA therapy may be the best correlate of nephrotoxicity and that the maintenance dose contributes little to chronic renal damage.

The relationship of hypertension and nephrotoxicity in CsA therapy is of particular interest. Nephrotoxicity was significantly worse in the four patients who developed hypertension requiring treatment during CsA therapy and in one patient with controlled hypertension prior to CsA treatment. Two patients who developed hypertension had received the higher induction dose of $10 \mathrm{mg} /$ $\mathrm{kg} /$ dayy and also had the longest duration of maintenance treatment. Hypertension frequently accompanies CsA nephrotoxicity, both in transplant and non-transplant patients ${ }^{9,18-20}$ and although hypertension has been stated to occur in patients with normal renal function, ${ }^{21}$ there is little published data to support this statement since in most reports of CsA' associated hypertension, the mean serum creatinine concentration is elevated. In renal transplant recipients, both hypertension and renal function have been reported to improve upon conversion from CsA therapy to combined prednisolone and azathioprine. ${ }^{19,20}$ It has been suggested in other studies of CsA treated eye disease that hypertension is more frequent in patients receiving combined $\mathrm{CsA}$ and corticosteroid therapy than CsA alone. ${ }^{9,22}$ This detrimental effect on blood pressure may nullify any benefits accruing from a lower maintenance dose of CsA, and may help explain the similar incidence of nephrotoxicity and hypertension in this study as in other studies employing a maintenance dose of $10 \mathrm{mg} / \mathrm{kg} / \mathrm{day}$.
Hypertension and nephrotoxicity in CsA treated patients are probably due to several factors, but recent experimental and clinical studies have suggested that changes in renal vascular resistance may be particularly important. Nifedipine, a calcium channel blocker with vasodilatory and hypotensive properties, has been shown in CsA treated renal transplant patients with stable renal function to result in improvement in glomerular ${ }^{23}$ and tubular ${ }^{24}$ parameters. The elucidation of the link between hypertension and nephrotoxicity is clinically important since it may offer a means of ameliorating CsA induced nephrotoxicity and help determine the most appropriate antihypertensive therapy for these patients.

It is interesting to note that, in this study, none of the younger patients ( $<35$ years) developed any serious toxicity from CsA therapy and all have shown a favourable clinical response to treatment. This must not allow us to become complacent about the side effects of chronic CsA treatment, and investigation into the mechanisms of and means of minimising toxicity are as necessary now as when this valuable drug was first intoduced ten years ago.

We wish to thank Professor W. Foulds, Dr. S. Cant, Dr. W. Haining, Dr. J. Coleiro, D. J. Cormack, and Dr. I. Hunter for kindly referring patients to Aberdeen for consideration of Cyclosporin A therapy.

\section{References}

${ }^{1}$ Forrester JV: Chronic Intraocular Inflammation. Trans Ophthalmol Soc UK 1985, 104: 250-5.

${ }^{2}$ Department of Health and Social Security: Blindness and partial sight in England 1969-1976. London: HMSO, 1979. (Reports on Public Health and Medical Subjects No. 129.)

${ }^{3}$ Nussenblatt RB, Palestine AG, Rook AH, et al.: Treatment of intraocular inflammatory disease with Cyclosporin A. Lancet 1983, ii: 235-38.

${ }^{4}$ Nussenblatt RB, Rodrigues MM, Walker WB, et al. : Cyclosporin A. Inhibition of experimental autoimmune uveitis in Lewis rats. J Clin Invest 1981, 67: $1228-31$

${ }^{5}$ Nussenblatt RB, Palestine AG, Chan CC: Cyclosporin A therapy in the treatment of intraocular inflammatory disease resistant to systemic corticosteroids and cytotoxic agents. Am J Ophthalmol 1983, 96: 275-82.

${ }^{6}$ Binder AI, Graham EM, Sanders MD, Dinning W, James DG, Denman AM: Cyclosporin A in the treatment of severe Behçet's uveitis. Br J Rheumatol 1987, 26: 285-91.

${ }^{7}$ Muftuoglu AU, Pazarli H, Yurdakul S, et al : Short 
term cyclosporin A treatment of Behçet's disease. Br J Ophthalmol 1987, 71: 387-90.

${ }^{8}$ Masuda K, Nakajima A, Urayama A, et al.: Doublemasked trial of Cyclosporin versus Colchicine and long-term study of Cyclosporin in Behçet's Disease. Lancet 1989, i: 1093-6.

${ }^{9}$ Palestine AG, Nusenblatt RB, Chan CC: Side effects of systemic cyclosporin in patients not undergoing transplantation. Am J Med 1984, 77: 652-6.

${ }^{10}$ Palestine AG, Austin HA, Balow JE, et al.: Renal histopathological alterations in patients treated with cyclosporine for uveitis. N Eng J Med 1986, 314: 1293-8.

${ }^{11}$ Klintmalm G, Bohman S, Sundelin B, Wilczek, H: Interstitial fibrosis in renal allografts after 12 to 46 months of cyclosporin treatment: beneficial effects of low doses in early post-transplantation period. Lancet 1984, ii: 950-4.

${ }^{12}$ Svenson K, Bohman S-O, Hallgren R: Renal interstitial fibrosis and vascular changes. Occurrence in patients with autoimmune diseases treated with Cyclosporine. Arch Int Med 1986, 146: 2007-10.

${ }^{13}$ Starzl TE, Nalesnik NA, Porter KE, et al.: Reversibility of lymphomas and lymphoproliferative lesions developing under cyclosporine-steroid therapy. Lancet 1984, ii: 583-7.

${ }^{14}$ Towler HM, Cliffe AM, Whiting PH, Forrester JV: Low dose Cyclosporin A therapy in chronic posterior uveitis. Eye 1989, 3: 282-7.

${ }^{15}$ Nussenblatt RB, Palestine AG, Chan CC, Roberge F: Standardization of vitreal inflammatory activity in intermediate and posterior uveitis. Ophthalmology 1985, 92: 467-71.
${ }^{16}$ Thomsen K: Lithium clearance: a new method for determining proximal and distal tubular resorption of sodium and water. Nephron 1984, 37: 217-23.

${ }^{17}$ Palestine AG, Austin AA, Nussenblatt RB: Renal tubular function in Cyclosporine-treated patients. Am J Med 1986, 81: 419-24.

${ }^{18}$ Deray G, Le Hoang P, Cacoub P, et al.: Renal function and blood pressure in patients treated with Cyclosporin A for uveitis. Eur J Clin Pharmacol 1988, 34: 601-4.

${ }^{19}$ Hamilton DV, Carmichael DJS, Evans DB, Calne RY: Hypertension in renal transplant recipients on Cyclosporin A and corticosteroids and azathioprine. Transpl Proc 1982, 14: 597-600.

${ }^{20}$ Chapman JR, Marcen R, Arias M, et al.: Hypertension after renal transplantation. A comparison of cyclosporine and conventional immunosuppression. Transplantation 1987, 43: 860-4.

${ }^{21}$ Leading Article: Cyclosporin hypertension. Lancet 1988, ii: 1234.

${ }^{22}$ Kahaly G, Schrezenmeir J, Krause U, et al.: Cyclosporin and prednisone $\mathbf{v}$. prednisone in treatment of Graves' ophthalmopathy: a controlled, randomised and prospective study. Eur J Clin Invest 1986, 16: 415-22.

${ }^{23}$ Mcnally PG, Feehally J, Walls J: Nifedipine increases GFR in chronic Cyclosporin-treated renal transplant recipients. Nephr Dialysis transpl 1989, 4: 515 (abstract).

${ }^{24}$ Propper DJ, Whiting PH, Power DA, et al.: The effect of nifedipine on graft function in renal àlograft recipients treated with Cyclosporin A Clin Nephrol (in press). 